\title{
Front Matter: Volume 9913
}

, "Front Matter: Volume 9913," Proc. SPIE 9913, Software and Cyberinfrastructure for Astronomy IV, 991301 (4 November 2016); doi: $10.1117 / 12.2254988$

Event: SPIE Astronomical Telescopes + Instrumentation, 2016, Edinburgh, SPIE. United Kingdom 


\title{
PROCEEDINGS OF SPIE
}

\section{Software and Cyberinfrastructure for Astronomy IV}

\author{
Gianluca Chiozzi \\ Juan C. Guzman \\ Editors
}

26-30 June 2016

Edinburgh, United Kingdom

Sponsored by

SPIE

Cooperating Organizations

American Astronomical Society (United States) - Australian Astronomical Observatory (Australia) - Association of Universities for Research in Astronomy (AURA) - Canadian Astronomical Society (CASCA) (Canada) - Canadian Space Agency (Canada) • European Astronomical Society (Switzerland) • European Southern Observatory (Germany) • National Radio Astronomy Observatory - Royal Astronomical Society (United Kingdom) • Science \& Technology Facilities Council (United Kingdom)

Published by

SPIE

Volume 9913

Proceedings of SPIE 0277-786X, V. 9913

SPIE is an international society advancing an interdisciplinary approach to the science and application of light.

Software and Cyberinfrastructure for Astronomy IV, edited by Gianluca Chiozzi,

Juan C. Guzman, Proc. of SPIE Vol. 9913, 991301 - C) 2016 SPIE

CCC code: $0277-786 \mathrm{X} / 16 / \$ 18 \cdot$ doi: $10.1117 / 12.2254988$

Proc. of SPIE Vol. $9913991301-1$

Downloaded From: https://www.spiedigitallibrary.org/conference-proceedings-of-spie on $26 \mathrm{Apr} 2023$ Terms of Use: https://www.spiedigitallibrary.org/terms-of-use 
The papers in this volume were part of the technical conference cited on the cover and title page. Papers were selected and subject to review by the editors and conference program committee. Some conference presentations may not be available for publication. Additional papers and presentation recordings may be available online in the SPIE Digital Library at SPIEDigitallibrary.org.

The papers reflect the work and thoughts of the authors and are published herein as submitted. The publisher is not responsible for the validity of the information or for any outcomes resulting from reliance thereon.

Please use the following format to cite material from these proceedings:

Author(s), "Title of Paper," in Software and Cyberinfrastructure for Astronomy IV, edited by Gianluca Chiozzi, Juan C. Guzman, Proceedings of SPIE Vol. 9913 (SPIE, Bellingham, WA, 2016) Sixdigit Article CID Number.

ISSN: 0277-786X

ISBN: 9781510602069 (electronic)

ISBN: 9781510602052

Published by

SPIE

P.O. Box 10, Bellingham, Washington 98227-0010 USA

Telephone +1 3606763290 (Pacific Time) · Fax +1 3606471445

SPIE.org

Copyright @ 2016 , Society of Photo-Optical Instrumentation Engineers.

Copying of material in this book for internal or personal use, or for the internal or personal use of specific clients, beyond the fair use provisions granted by the U.S. Copyright Law is authorized by SPIE subject to payment of copying fees. The Transactional Reporting Service base fee for this volume is $\$ 18.00$ per article (or portion thereof), which should be paid directly to the Copyright Clearance Center (CCC), 222 Rosewood Drive, Danvers, MA 01923. Payment may also be made electronically through CCC Online at copyright.com. Other copying for republication, resale, advertising or promotion, or any form of systematic or multiple reproduction of any material in this book is prohibited except with permission in writing from the publisher. The CCC fee code is 0277-786X/16/\$18.00.

Printed in the United States of America.

Publication of record for individual papers is online in the SPIE Digital Library.

\section{SPIE. DIGITAL}

Paper Numbering: Proceedings of SPIE follow an e-First publication model. A unique citation identifier (CID) number is assigned to each article at the time of publication. Utilization of CIDs allows articles to be fully citable as soon as they are published online, and connects the same identifier to all online and print versions of the publication. SPIE uses a six-digit CID article numbering system structured as follows:

- The first four digits correspond to the SPIE volume number.

- The last two digits indicate publication order within the volume using a Base 36 numbering system employing both numerals and letters. These two-number sets start with 00, 01, 02, 03, 04, $05,06,07,08,09,0 A, 0 B \ldots$. OZ, followed by 10-1Z, 20-2Z, etc. The CID Number appears on each page of the manuscript. 


\title{
Contents
}

\author{
xiii Authors \\ xxi Conference Committee
}

\section{Part One}

\section{SESSION 1 PROJECT OVERVIEWS AND PROGRESS I}

991302 SKA Telescope Manager (TM): status and architecture overview (Invited Paper) [9913-1]

991303 The software architecture to control the Cherenkov Telescope Array [9913-2]

991304 The transition from construction to operations on the ALMA control software [9913-3]

\section{SESSION 2 TELESCOPE CONTROL I}

991305 Improving the pointing and tracking performance of the Keck telescopes [9913-106]

991306 The active surface control system for the Tian Ma Telescope [9913-6]

991307 Computer-aided star pattern recognition with astrometry.net: In-flight support of telescope operations on SOFIA [9913-7]

991308 Control and monitoring software for the Greenland Telescope [9913-8]

991309 LSST control software component design [9913-9]

SESSION 3 SOFTWARE QUALITY AND TESTING

9913 OA An automated qualification framework for the MeerKAT CAM (Control-And-Monitoring) [9913-10]

$9913 \mathrm{OB} \quad$ Rules of thumb to increase the software quality through testing [9913-11]

9913 OC Behavior driven testing in ALMA telescope calibration software [9913-12]

9913 OD The evolution of the simulation environment in the ALMA Observatory [9913-13]

9913 OE Modernized build and test infrastructure for control software at ESO: highly flexible building, testing, and automatic quality practices for telescope control software [9913-14] 
9913 OF High-contrast imaging in the cloud with klipReduce and Findr [9913-134]

9913 OG Investigating interoperability of the LSST data management software stack with Astropy [9913-16]

$9913 \mathrm{OH} \quad$ VIALACTEA knowledge base homogenizing access to Milky Way data [9913-17]

9913 Ol A case study in adaptable and reusable infrastructure at the Keck Observatory Archive: VO interfaces, moving targets, and more [9913-18]

\section{SESSION 5 CYBERINFRASTRUCTURE}

9913 OK A cyber infrastructure for the SKA Telescope Manager [9913-20]

9913 OL The NOAO data lab: science-driven development [9913-128]

9913 OM The AST3 controlling and operating software suite for automatic sky survey [9913-22]

9913 ON TMT common software update [9913-23]

991300 DDS as middleware of the Southern African Large Telescope control system [9913-24]

\section{SESSION $6 \quad$ INSTRUMENTATION CONTROL}

9913 OP The DESI instrument control system [9913-25]

$99130 Q \quad$ Efficient receiver tuning using differential evolution strategies [9913-26]

9913 OR The South African Astronomical Observatory instrumentation software architecture and the SHOC instruments [9913-27]

9913 OS World coordinate information for the Daniel K. Inouye Solar Telescope [9913-28]

9913 OT The Infrared Imaging Spectrograph (IRIS) for TMT: motion planning with collision avoidance for the on-instrument wavefront sensors [9913-29]

9913 OU AAO Starbugs: software control and associated algorithms [9913-30]

9913 OV Collision-free coordination of fiber positioners in multi-object spectrographs [9913-31]

\section{SESSION 7 UI/WEB TECHNOLOGIES}

9913 OW Exploratory visualization of astronomical data on ultra-high-resolution wall displays (Invited Paper) [9913-32] 
9913 0X Prototyping the graphical user interface for the operator of the Cherenkov Telescope Array [9913-33]

9913 OY Firefly: embracing future web technologies [9913-34]

$99130 Z$ Observation management challenges of the Square Kilometre Array [9913-35]

SESSION 8 PROJECT OVERVIEWS AND PROGRESS II

991310 Status report of the SRT radiotelescope control software: the DISCOS project [9913-36]

991311 Status report of the end-to-end ASKAP software system: towards early science operations [9913-37]

991312 MAISIE: a multipurpose astronomical instrument simulator environment [9913-38]

991313 ACS from development to operations [9913-39]

991314 The ESO astronomical site monitor upgrade [9913-40]

SESSION 9 DATA PROCESSING AND PIPELINES I

991315 ASTRI SST-2M prototype and mini-array data reconstruction and scientific analysis software in the framework of the Cherenkov Telescope Array [9913-41]

991316 Implementing a real-time data stream for time-series stellar photometry [9913-42]

991317 Automated spectral reduction pipelines [9913-43]

991318 StarDock: shipping customized computing environments to the data [9913-44]

991319 TMT approach to observatory software development process (Invited Paper) [9913-45]

$99131 \mathrm{~A}$ Don't get taken by surprise: planning for software obsolescence management at the ALMA Observatory [9913-46]

9913 1B Management of the science ground segment for the Euclid mission [9913-47]

9913 1D Building a world-wide open source community around a software framework: progress, dos, and don'ts [9913-49] 


\section{Part Two}

\section{SESSION 11}

DATA MANAGEMENT AND ARCHIVES II

9913 IE The new Gemini Observatory archive: a fast and low cost observatory data archive running in the cloud [9913-50]

9913 IF Petascale cyberinfrastructure for ground-based solar physics: approach of the DKIST data center [9913-51]

9913 IG Cloud services on an astronomy data center [9913-119]

9913 1H Trident: scalable compute archives: workflows, visualization, and analysis [9913-53]

991311 The NOAO Data Lab virtual storage system [9913-54]

\section{SESSION 12 TELESCOPE CONTROL II}

$99131 \mathrm{~J} \mathrm{Mount} \mathrm{control} \mathrm{system} \mathrm{of} \mathrm{the} \mathrm{ASTRI} \mathrm{SST-2M} \mathrm{prototype} \mathrm{for} \mathrm{the} \mathrm{Cherenkov} \mathrm{Telescope} \mathrm{Array}$ [9913-55]

$99131 \mathrm{~K}$ Automation and control of the MMT thermal system [9913-56]

$99131 \mathrm{~L}$ Software architecture of INO340 telescope control system [9913-57]

$99131 \mathrm{M}$ Introduction to FAST central control system [9913-58]

9913 IN Prototyping the E-ELT M1 local control system communication infrastructure [9913-59]

991310 A new telescope control software for the Mayall 4-meter telescope [9913-60]

\section{SESSION 13 SOFTWARE ENGINEERING}

9913 IP Revisiting software specification and design for large astronomy projects (Invited Paper) [9913-61]

$99131 Q$ Software requirements flow-down and preliminary software design for the G-CLEF spectrograph [9913-62]

$99131 R \quad$ Software framework for automatic learning of telescope operation [9913-63]

9913 is Can your software engineer program your PLC? [9913-64]

SESSION 14 DATA PROCESSING AND PIPELINES II

$99131 \mathrm{~T}$ Integrated data analysis in the age of precision spectroscopy: the ESPRESSO case [9913-66] 
$99131 \mathrm{U}$ RabbitQR: fast and flexible big data processing at LSST data rates using existing, shareduse hardware [9913-67]

9913 IV GAVIP: a platform for Gaia data analysis [9913-68]

POSTER SESSION: CYBERINFRASTRUCTURE, HIGH-PERFORMANCE AND PARALLEL COMPUTING, BIG DATA

$99131 \mathrm{~W} \quad$ Is the work flow model a suitable candidate for an observatory supervisory control infrastructure? [9913-21]

$99131 \mathrm{X}$ WAS: the data archive for the WEAVE spectrograph [9913-71]

$99131 Y$ The very high energy source catalog at the ASI Science Data Center [9913-76]

991312 Telemetry correlation and visualization at the Large Binocular Telescope Observatory [9913-79]

991320 The ALMA high speed optical communication link is here: an essential component for reliable present and future operations [9913-83]

991323 Operational logs analysis at ALMA observatory based on ELK stack [9913-95]

991324 The Open Microscopy Environment: open image informatics for the biological sciences [9913-99]

991326 Advanced GLS map-making for the Herschel's photometers [9913-107]

991327 Data reduction software for the Mid-Infrared E-ELT Imager and Spectrograph (METIS) for the European Extremely Large Telescope (E-ELT) [9913-110]

991328 A distributed infrastructure for publishing VO services: an implementation [9913-116]

9913 2A The HARPS-N archive through a Cassandra, NoSQL database suite? [9913-125]

9913 2B Virtualizing observation computing infrastructure at Subaru Telescope [9913-131]

9913 2C Information and Communications Technology (ICT) Infrastructure for the ASTRI SST-2M telescope prototype for the Cherenkov Telescope Array [9913-137]

9913 2D Radio data archiving system [9913-182]

POSTER SESSION: OBSERVATORY, TELESCOPE AND INSTRUMENTATION CONTROL

$99132 \mathrm{E} \quad$ Pre-selecting muon events in the camera server of the ASTRI telescopes for the Cherenkov Telescope Array [9913-70]

$99132 \mathrm{~F} \quad$ Automatization of the guiding process in the GTC [9913-74]

$99132 \mathrm{G}$ Wendelstein Observatory control software [9913-78] 
$99132 \mathrm{H} \quad$ Integration of the instrument control electronics for the ESPRESSO spectrograph at ESO-VLT [9913-82]

$991321 \quad$ The ICT monitoring system of the ASTRI SST-2M prototype proposed for the Cherenkov Telescope Array [9913-86]

$99132 \mathrm{~J} \quad$ Challenges and strategies for the maintenance of the SKA Telescope Manager [9913-90]

$99132 \mathrm{~K}$ The technical CCDs in ESPRESSO: usage, performances, and network requirements [9913-94]

$99132 \mathrm{~L}$ The SKA observation control system [9913-98]

$99132 \mathrm{M}$ Rejecting harmonic vibrations at Gemini with real-time vibration tracking [9913-102]

$991320 \quad$ LSST OCS status and plans [9913-112]

$99132 \mathrm{P}$ Target allocation and prioritized motion planning for MIRADAS probe arms [9913-115]

$99132 \mathrm{Q}$ GHOST and GIAPI: experience using Gemini's new instrument control system framework [9913-1 18]

$99132 \mathrm{R} \quad$ Using muon rings for the optical calibration of the ASTRI telescopes for the Cherenkov Telescope Array [9913-121]

9913 2T INO340 telescope mount control system analysis and design [9913-127]

$99132 \mathrm{U}$ Status, upgrades, and advances of RTS2: the open source astronomical observatory manager [9913-130]

$99132 \mathrm{~V} \quad$ Using Robotic Operating System (ROS) to control autonomous observatories [9913-133]

9913 2W On-board target acquisition for CHEOPS [9913-136]

$99132 X \quad$ Towards integrated modelling: full image simulations for WEAVE [9913-139]

$99132 Z$ SKA CSP controls: technological challenges [9913-141]

991330 Remote observing environment using a KVM-over-IP for the OAO $188 \mathrm{~cm}$ telescope [9913-143]

991331 The Cherenkov Telescope Array Observatory: top level use cases [9913-144]

991332 Remote operations at UKIRT, Cassegrain included, 2 years later [9913-145]

991333 Agile development approach for the observatory control software of the DAG 4m telescope [9913-147]

991334 The CARMENES instrument control software suite [9913-149]

991335 Target-based fiber assignment for large survey spectrographs [9913-151] 
991336 The 4MOST facility control software [9913-153]

$991337 \quad$ Modified deformable mirror stroke minimization control for direct imaging of exoplanets [9913-156]

991338 The instrument control software package for the Habitable-Zone Planet Finder spectrometer [9913-158]

\section{Part Three}

\section{POSTER SESSION: PROJECT OVERVIEWS AND PROGRESS REPORTS}

991339 Development of a real-time data processing system for a prototype of the Tomo-e Gozen wide field CMOS camera [9913-75]

9913 3A A reorganization cyberinfrastructure of history observing data in China [9913-84]

9913 3B The survey operation software system development for Prime Focus Spectrograph (PFS) on Subaru Telescope [9913-88]

9913 3C Status of the array control and data acquisition system for the Cherenkov Telescope Array [9913-92]

$99133 \mathrm{E} \quad$ The TESS science processing operations center [9913-100]

POSTER SESSION: SOFTWARE ENGINEERING, DESIGN, AND IMPLEMENTATION

991336 Software design of the ASTRI camera server proposed for the Cherenkov Telescope Array [9913-69]

9913 3I SINBAD flight software, the on-board software of NOMAD in ExoMars 2016 [9913-81]

9913 3J Porting the ALMA Correlator Data Processor from hard real-time to plain Linux [9913-85]

9913 3K Implementing the concurrent operation of sub-arrays in the ALMA correlator [9913-89]

9913 3L The ASTRI mini-array software system (MASS) implementation: a proposal for the Cherenkov Telescope Array [9913-93]

$99133 \mathrm{M}$ Concept study of an observation preparation tool for MICADO [9913-97]

991330 A new generation of spectral extraction and analysis package for Fiber Optics Cassegrain Echelle Spectrograph (FOCES) [9913-105]

9913 3P Monitoring service for the Gran Telescopio Canarias control system [9913-108]

$99133 Q \quad$ ESPRESSO front end guiding algorithms: from design phase to implementation and validation toward the commissioning [9913-1111] 
9913 3R EELT-HIRES the high resolution spectrograph for the E-ELT: software and hardware solutions for its control [9913-114]

991335 Monitoring and controlling the SKA telescope manager: a peculiar LMC system in the framework of the SKA LMCs [9913-117]

9913 3T The software architecture of the camera for the ASTRI SST-2M prototype for the Cherenkov Telescope Array [9913-120]

$99133 \mathrm{U}$ INO340 telescope control system: middleware requirements, design, and evaluation [9913-129]

$99133 \mathrm{~V}$ ASTRI SST-2M data reduction and reconstruction software on low-power and parallel architectures [9913-132]

9913 3W Towards a dynamical scheduler for ALMA: a science - software collaboration [9913-135]

$99133 \mathrm{X}$ Software design and code generation for the engineering graphical user interface of the ASTRI SST-2M prototype for the Cherenkov Telescope Array [9913-138]

991332 A real-time prediction system for solar weather based on magnetic nonpotentiality (I) [9913-146]

991340 Software use cases to elicit the software requirements analysis within the ASTRI project [9913-148]

$991341 \quad$ M-and-C Domain Map Maker: an environment complimenting MDE with M-and-C knowledge and ensuring solution completeness [9913-150]

991342 The RTE inversion on FPGA aboard the solar orbiter PHI instrument [9913-152]

991343 Knowledge-based engineering of a PLC controlled telescope [9913-154]

991344 Aided generation of search interfaces to astronomical archives [9913-157]

991346 A control system framework for the Hobby-Eberly telescope [9913-160]

991347 A user interface framework for the Square Kilometre Array: concepts and responsibilities [9913-162]

991349 Queve software reuse and implementation at the Large Binocular Telescope Observatory [9913-164]

9913 4A The Infrared Imaging Spectrograph (IRIS) for TMT: data reduction system [9913-165]

9913 4C Key software architecture decisions for the automated planet finder [9913-167]

9913 4D AVU/BAM: software refurbishment (design and implementation) for the CU3 Gaia verification pipeline [9913-168]

9913 4E ImageX: new and improved image explorer for astronomical images and beyond [9913-169] 
9913 4F The ExoMars DREAMS scientific data archive [9913-170]

9913 4G Image processing improvement for optical observations of space debris with the TAROT telescopes [9913-171]

$99134 \mathrm{~J}$ Thirty Meter Telescope (TMT) Narrow Field Infrared Adaptive Optics System (NFIRAOS) realtime controller preliminary architecture [9913-174]

$99134 \mathrm{~K} \quad$ FRIDA's mechanisms control system structure and tests [9913-175]

$99134 \mathrm{~L}$ A virtual appliance as proxy pipeline for the Solar Orbiter/Metis coronagraph [9913-176]

9913 4M The ALMA Snooping Project Interface (SnooPI) [9913-177]

$99134 \mathrm{~N}$ Observatory software for the Maunakea Spectroscopic Explorer [9913-178]

991340 Synchronization of off-centered dome and 3.6m Devasthal Optical Telescope [9913-179]

9913 4P A novel approach to visual rendering of astro-photographs [9913-180] 
Proc. of SPIE Vol. $9913991301-12$

Downloaded From: https://www.spiedigitallibrary.org/conference-proceedings-of-spie on 26 Apr 2023 Terms of Use: https://www.spiedigitallibrary.org/terms-of-use 


\section{Authors}

Numbers in the index correspond to the last two digits of the six-digit citation identifier (CID) article numbering system used in Proceedings of SPIE. The first four digits reflect the volume number. Base 36 numbering is employed for the last two digits and indicates the order of articles within the volume. Numbers start with 00, 01, 02, 03, 04, 05, 06, 07, 08, 09, 0A, 0B...0Z, followed by 10-1Z, 20-2Z, etc.

Abareshi, Behzad, 10

Aboudan, A., 4F

Abrams, Don Carlos, 1X, 2X

Abril, M., 34

Alberti, Valentina, 47

Aliverti, M., 3Q

Allan, Chris, 24

Allen, S. L., 4C

Altieri, Bruno, 26

Álvarez, C., 2F

Amado, P. J., 34

Amestica, Rodrigo, 3J, 3K

Anderson, Tyler, 38

Andolfato, L., $1 \mathrm{~N}$

Andretta, V., 4L

Antognini, Jonathan, 1D

Antolini, Elisa, 1J, 3L, 3X, 40

Antonelli, Lucio Angelo, 15, 1Y, 31, 3L, 3V

Antonino, P. Oliveira, 03

Antonucci, E., 4L

Aoki, Tsutomu, 39

Aparicio-del-Moral, Beatriz, 31, 42

Apestigue-Palacio, $V_{\text {., }} 4 \mathrm{~F}$

Appert, Caroline, OW

Araya, Mauricio, $1 G$

Argomedo, J., 1N

Arimatsu, Ko, 39

Arruego-Rodríguez, I., 4F

Artigues, Gabriel, 2V

Astier, Joseph, 49

Astudillo, A., 20

Avarias, Jorge, 1D, 3W

Baccani, C., 4L

Bacigalupo, Carlos, OU

Baffa, C., $2 Z$

Bagaglia, Marco, $1 \mathrm{~J}$

Bagano, Cy, 2M

Balaguer, M., 42

Baldini, V., 2H, 2K, 3R

Balzer, Arnim, 3C

Bandieramonte, Marilena, $\mathrm{OH}$

Banerjee, Amar, 41

Barbosa, Domingos, 02, OK

Barnsley, Robert M., 17

Barraca, João Paulo, 02, 0K

Barriga, $\mathrm{P} ., 1 \mathrm{~N}$

Bartolini, M., OB, 10

Bastieri, Denis, 15, 3V

Beard, Steven, 12
Beaufore, L., OP

Becciani, Ugo, $\mathrm{OH}$

Bedosti, F., 2D

Bellassai, G., 2C

Bender, Chad F., 38

Bender, Ralf, 30

Benítez, D., 34

Benn, Chris, $2 X$

Berge, David, 3C

Berkenbosch, Sophie, 31

Berriman, G. Bruce, 0 I

Berthold, Ryan M., 32

Berukoff, Steven, 1F, 1P

Besson, Sébastien, 24

Bettanini, C., 4F

Bhatia, Ravinder, 19

Biddick, Christopher, $1 \mathrm{Z}$

Bierwirth, Thomas, 14

Bignamini, Andrea, 44

Bigongiari, Ciro, 31, 3L

Blackburn, Colin, 24

Blanchet, G., 4G

Boër, M., 4G

Bogosavljevic, M., 16

Boisson, C., 31

Bolton, Adam S., OL, 11

Bonanomi, C., 4P

Bonifacio, Piercarlo, 1X, 2X

Bonnet, Henri, 2M

Bonnewijn, Sabrina, 31

Bonnoli, Giacomo, 40

Borkowski, Jerzy, 3C

Borrowman, Alastair J., is

Bosch, James, OG

Bosnjak, Z., 31

Bovill, Robert, 09

Boyer, Corinne, 4J

Brajnik, Giorgio, 47

Brandl, Bernhard R., 27

Brau-Nogué, S., 31

Brescia, Massimo, $\mathrm{OH}$

Bridger, Alan, 02, 0Z, 2L

Brighton, Allan, $\mathrm{ON}$

Brink, Janus D., 00

Brito, H., 2F

Broguiere, Dominique, OC

Brucalassi, Anna, 30

Bruno, Pietro, 2C, 2I, 3L, 3X, 40

Bryant, Randy, 46 
Buckley-Geer, E., OP

Budynkiewicz, Jamie A., 1Q

Buenadicha, Guillermo, 1B

Bulgarelli, Andrea, 31, 3G, 3L, 40

Burel, Jean-Marie, 24

Busatta, Andrea, $1 \mathrm{~J}$

Butler, Karen, 10

Butora, Robert, $\mathrm{OH}$

Buttu, M., OB, 10

Buur, Hanne, ON, 19

Buzzi, Raffaella, 4D

Caballero, J. A., 34

Cabral, A., 3Q

Calderone, Giorgio, 1T, 2H, 2K, 3Q, 3R

Caldwell, Douglas A., 3E

Calzoletti, Luca, 26

Campbell, Jennifer, 3E

Candini, Gian Paolo, 31

Canestrari, Rodolfo, 1J, 3L, 3X

Cantarutti, Rolando, 10

Canzari, Matteo, 35

Capalbi, Milvia, 2E, 3G, 3L, 3T, 40

Caproni, Alessandro, 13, 1D

Cardenes, Ricardo, $1 \mathrm{E}$

Cardwell, Andrew, 49

Carosi, Alessandro, 1Y, 31

Carr, R., 4L

Carrasco Licea, Esperanza E., 1X, 2X

Carroll, Mark, 24

Carvalho, Bruno, OK

Cascone, Enrico, 1 J, 3L

Castander, F., OP

Catalano, Osvaldo, 3G, 3L, 3T

Cavedoni, Chas P., 2M

Cavuoti, Stefano, $\mathrm{OH}$

Cepparo, Francesco, 28, 44

Chacon, A. Dean, 3E

Chandrasekharan, Srinivasan, 09

Chapin, Edward L., OT, 4A

Chapman, Jessica, 11

Chapuis, Olivier, OW

Chavan, A. Maurizio, 4M

Chen, A., 31

Chiozzi, Gianluca, 13, 14

Choudhury, Subhrojyoti Roy, 02, 2L, 41

Ciardi, D., OY

Cirami, Roberto, 1T, 2H, 2K, 3Q, 3R

Clairquin, Roland, 3 I

Close, Laird M., OF

Cobos Carrascosa, J. P., 42

Coffaro, Martina, 2E

Cohen, Richard W., 0 I

Çoker, Deniz, 33

Colombatti, G., 4F

Colomé, Josep, 03, 2V, 31, 34, 3C

Colomer, $\mathrm{Pau}, 13$

Colson, Andrew, 0

Conforti, Vito, 03, 2C, 2E, 2I, 31, 3C, 3G, 3L, 3X, 40

Contaxis, Christopher, 09

Contreras, J. L., 31
Cookson, Jamie L, 32

Coretti, I., 2H, 2K, 3R

Costa, Alessandro, $\mathrm{OH}$

Cotter, G., 31

Covino, S., 31

Cristiani, Stefano, 1T, 2H, 2K, 3Q, 3R

Cuevas, S., 4K

Cumming, Tom, 2M

Cupani, Guido, 1T, 3R

Cusumano, Giancarlo, 2R

Czoske, Oliver, 27

Dabin, Christophe, 1B

daCosta, L., OP

Dalton, Gavin B., 1X, 2X

Daly, Philip N., 09, 1W, 20

Daniel, M., 31

De Cesare, G., 31

de Groof, A., 4L

de Guindos, E., 34

de Juan, E., 34

de Ona Wilhelmi, E., 31

de Paz, H., 2F

de Val-Borro, Miguel, OG

Debei, $S$., $4 \mathrm{~F}$

Deconinck, Geert, 43

Deich, William T. S., 4C

del Campo, Fernando, OW

del Toro Iniesta, J. C., 42

Del Valle, Diego, 14

Delanoye, Sofie, 31

Delgado, Francisco, 09, 20

Della Volpe, M., 31

Deng, Yuanyong, 3A, $3 Z$

DePonte Evans, Janet, $1 Q$

Dey, Arjun, 10

Di Carlo, Matteo, 02, 2J, 3S

Di Giorgio, Anna M., OH

Di Marcantonio, Paolo, 1T, 2H, 2K, 3Q, 3R

Di Michele, Daniele, $1 \mathrm{~J}$

Di Paola, Andrea, 3L

Di Pierro, Federico, 31, 3L

Díaz, J. J., 4K

Dimmler, M., IN

Ding, Minde, $3 A$

Do, Tuan, 4A

D'Odorico, Valentina, 1T, 3R

Doi, Mamoru, 39

Dolci, Mauro, 02, 2J, 35

Dole, Hervé, OW

Dong, Jian, 06

Donnarumma, Imma, 15

Dorigo, Dario, 14

Dovgan, E., 2D

Drimmel, Ronald, 4D

Drory, Niv, 46

Drummond, Rachel, 3 I

Du, Zhangle, 3A

Dubois-Felsmann, Gregory P., 09, 0G, OY

Dumas, Christophe, 19

Dunn, Jennifer, OT, 4A, 4J 
Edwards, Michelle L., 49

Eldorado Riggs, A. J., 37

Elia, Davide, $\mathrm{OH}$

Ellerbroek, Brent, 4A, 4J

Elliott, A. E., OP

Elliott, Linda, 46

Espada, Daniel, 3W

Espejo, C., 4K

Esposito, F., 4F

Evans, Ian N., $1 Q$

Eyheramendy, Susana, OW

Fara, A., 10

Farias, Humberto, $1 \mathrm{G}$

Farrell, Tony J., OU

Fausti, A., OP

Ferguson, Richard K., 24

Ferstl, R., 2W

Feyrin, S., $\mathrm{OE}$

Filippi, G., 20

Fioretti, Valentina, 2I, 31, 3G, 3L, 40

Fitzpatrick, Michael J., OL, II

Flores-Meza, R., 4K

Flynn, Helen, 24

Focardi, M., 4L

Fowler, James, 46

Fresno, M. L., 34

Fu, Li, 06

Fuessling, Matthias, 03, 1D, 31, 3C

Fugazza, Dino, 40

Gabor, Hermann, $\mathrm{OH}$

Gaggstatter, T., 2F

Gai, Mario, 4D

Gallozzi, Stefano, 2C, 3 L

Gambini, Giorgio Francesco, 1 J, 3X

Garcés, J., 4K

Garces, Mario, OC

García-Piquer, Álvaro, 2V, 34

Garczarczyk, M., 31

Gardiol, Daniele, 3L, 40

Garzón, Francisco, 2P

Gaug, M., 31

Gault, David, 24

Geers, Vincent, 12

Gelino, Christopher R., Ol

Genoni, M., 3Q

Genzer, M., 4F

Gesa, LI., 34

Giacomel, Stefano, $1 \mathrm{~J}$

Giani, E., $2 Z$

Gianotti, Fulvio, 2C, 2I, 3G, 3L

Gibson, J. Duane, 1K

Gil, Juan Pablo, 0C, 23

Gillen, Kenneth, 24

Gilles, Luc, 4J

Gillet, Denis, OV

Gillies, Kim, ON, OT, 19, 4A

Gimenes, Renato, 3T

Gimenez, Guillermo, 1D

Giommi, Paolo, $1 Y$

Giro, Enrico, 3L
Girouard, Forrest, 3E

Glicenstein, J. F., 31

Goble, William, $1 \mathrm{~K}$

Goldina, T., OY

Goldoni, P., 31

Gómez, José María, 2P

Gómez-López, Juan M., 31

González-Hernández, Jonay I., IT

Good, John C., Ol

Goodrich, Bret, OS

Goodwin, Michael, OU

Gopinathan, Maheswar, 40

Gopu, Arvind, 18, 1H, 1U, 4E

Gössl, Claus, 2G

Gott, Shelby, 10

Göłz, D., 31

Goullon, J., 31

Graham, Matthew J., OL, 11

Grandi, P., 31

Gregorio, Anna, 1B

Greimel, Robert, 27

Griffith, Morgan, 1A

Grigolon, Carlo, $1 \mathrm{~J}$

Grillo, Alessandro, 2I, 3L, 40

Groff, Tyler, 37

Grudzien, T., 1N

Grupp, Frank, 30

Guàrdia, J., 34

Guarise, Paolo, $1 \mathrm{~J}$

Güçsav, B. Bülent, 33

Güdel, Manuel, 27

Guerra, Dailos, 1R, 2F

Guerra, Jose, 1X, 2A

Gupta, Yashwant, 02, 0K

Guzman, Juan Carlos, 02, 11

Hagen, H.-J., 34

Hagge, L., 03

Hahn, Thomas, 36

Hajnal, Akos, $\mathrm{OH}$

Halevin, Alexandros, 4M

Halverson, Samuel, 38

Ham, Sun Jeong, 2X

Hanlon, Lorraine, 1V

Harbeck, Daniel, $1 \mathrm{H}$

Hardash, Steve, $2 M$

Harri, A.-M., 4F

Harutyunyan, Avet, 2A

Hatziminaoglou, Evanthia, 4M

Haucke, J., OE

Haugan, S. V., 4L

Haug-Baltzell, Asher, OF

Hauschildt-Purves, Maren, 32

Hayano, Yutaka, OT, 4A

Hayashi, Soichi, 18, 1H, 1U, 4E

Hays, T., IF

Hayward, Thomas L., 2M

Hearty, Frederick, 38

Heiges, Cory, 3E

Heller, M., 31

Hempel, Maren, OW 
Henschel, Robert, $1 \mathrm{H}$

Henze, Christopher, 3E

Hermann, G., 31

Herriot, Glen, 4J

Hibbard, John, 3W

Hill, Gary J., 46

Hinton, J., 31

Hiriart, Rafael, 04, 3W

Hirst, Paul, 1E

Ho, Kevin, 4N

Hoar, John, 1B

Holden, Bradford P., 4C

Hong, Sungwook E., OU

Honscheid, K., OP

Hooper, Stephen, $1 Z$

Hopp, Ulrich, 30

$\mathrm{Hu}$, Keliang, OM

$\mathrm{Hu}, \mathrm{Yi}, \mathrm{OM}$

Huang, Chih-Wei Locutus, 08

Huang, Lijuan Wendy, OL, 11

Huertas, Manuel, 2F, 3P

Hümmer, Martin, 07

Ibsen, Amanda, OW

Ibsen, Jorge, OD, 1A, 1D, 20

Ichiki, Makoto, 39

Ikeda, Shiro, 39

Inagaki, Takeshi, 2B

Inove, Goki, 30

Inove, S., 31

loannou, Z., 16

Isani, Sidik, 4N

Ita, Yoshifusa, 39

Izumiura, Hideyuki, 30

Jafarzadeh, Asghar, 2T

Jangid, Purshottam, $4 \mathrm{O}$

Jaque, S., 20

Jenkins, Jon M., 3E

Jenness, Tim, 09, OG

Jeram, Bogdan, $\mathrm{OE}, 13$

Jerónimo-Zafra, Jose M., 31

Jerse, Giovanna, $0 Z$

Jeschke, Eric, 2B

Ji, Haisheng, 3A

Jiang, Zhiqian, $1 \mathrm{M}$

Jin, Huiliang, 06

Jin, Shoko, 1X, 2X

Johnson, Chris, OT, 4A

Johnson, Tony, 09

Joliet, E., OY

Jordan, Andres, OW

Joyce, Dick, 10

Kackley, Russell, 2B

Kacsuk, Peter, $\mathrm{OH}$

Kasdin, N. Jeremy, 37

Kasuga, Toshihiro, 39

Kausch, Wolfgang, 27

Kawakita, Hideo, 39

Keiman, C., 4K

Kellermann, Hanna, 30

Kent, S., OP
Kerley, Dan, 4J

Kerschbaum, F., 2W

Keskin, Onur, 33

Khelifi, B., 31

Khosroshahi, Habib, $1 \mathrm{~L}$

Kikuchi, Yuki, 39

Kirkby, D., OP

Klaassen, Pamela, 0Z, 12

Klaus, Todd, 3E

Klotz, A., 4G

Knapic, Cristina, OZ, 2D, 44

Kneib, Jean-Paul, OV, 35

Knödlseder, J., 31

Kobayashi, Naoto, 39

Kodric, Mihael, $2 \mathrm{G}$

Köhler, Rainer, 27

Kokubo, Mitsuru, 39

Kong, Mihseh, 01

Kornweibel, N., IN

Kosack, K., 31

Kosugi, George, 1A

Kotulla, Ralf, 1H, 1U, 4E

Krabbe, Alfred, 07

Krasuski, Tomas, 05

Krughoff, K. Simon, OG

Kubánek, Petr, $2 \mathrm{U}$

Kuehn, Kyler, OU

Kumar, Brijesh, $4 \mathrm{O}$

Kuroda, Daisuke, 30

Kwok, Shui Hung, 05

La Palombara, Nicola, 3L

La Parola, Valentina, 2R

La Rosa, Giovanni, 3T

Lacasse, Richard, 3K

Laity, Anastasia C., OI

Lanclos, Kyle, 4C

Landini, F., 4L

Landoni, M., 2K, 3Q

Landriau, Martin, 46

Lang, Dustin, 07

Lara, G., 4K

Larkin, James E., OT, 4A

Latham, David W., 3E

Lattanzi, Mario Gilberto, 4D

Le Roux, Gerhard, 02, 0K, 47

Leck, Ron, 46

Leese, Mark, 31

Leigh, Roger, 24

Leitzinger, Martin, 27

Lenain, J.-P., 31

Leo, Simone, 24

Leschinski, Kieran, 27

Leto, Giuseppe, 2C, 2I, 3L, 40

Levi, Eric, 38

Lewis, Jim, $2 X$

Li, Simon, 24

Licata, Enrico, 4D

Liello, F., 20

Lim, Kian-Tat, 09, 0G

Lin, Ganghua, 3A, 3 Z 
Lin, Jun, 3A

Lindemann, Rico, 3C

Lindfors, E., 31

Lindner, Dominik, 24

Linkert, Melissa, 24

Liu, Qinghui, 06

Liu, Scige J., $\mathrm{OH}$

Liu, Suo, 3A

Lodi, Marcello, 1X, 2A

Loeschl, P., 2W

Lombaard, Briehan, OR

Lombardi, Saverio, 15, 31, 3L, 3V, 3X, 40

Loomis, Craig P., 3B

López Aguerri, Jose Alfonso, 1X, 2X

López Jiménez, A. C., 42

López-Moreno, Jose J., 31

Lorente, Nuria P. F., OU

Lotz, Paul J., 09

Lovis, Christophe, $1 \mathrm{~T}$

Lucarelli, Fabrizio, 15, 1Y, 31, 40

Lung, David, 3E

Lupton, Robert H., OG, 3B

Luque-Escamilla, P., 31

Ly, L., OY

Lyard, Etienne, 03, 3C

Lyke, Jim, 05

Lynn, David, $1 \mathrm{~V}$

Lyons, Eric, OF

Ma, Bin, OM

Maartens, Deneys S., 00

Maccarone, Maria Concetta, 2E, 2R, 3L, 40

Mach, Michael, 27

Macías, Rosa, 2F, 3P

Mader, Jeffrey A., 05, 0l

Madonna, Alberto, 15, 3V

Maehara, Hiroyuki, 39

Maes, Jeroen, 31

Mahadevan, Suvrath, 38

Maia, Dalmiro, OK

Maier, G., 31

Makarem, Laleh, OV, 35

Malaguti, Giuseppe, 2C, 2I, 3G, 40

Males, Jared R., OF

Mañas, Miguel M., 13

Mandel, Holger, 36

Manfrin, Cristiana, $1 \mathrm{~J}$

Mannetta, M., 4F

Mansouri-Samani, Masoud, 3E

Mansutti, Oriana, 1B

Marais, Neilen, OA

Marassi, Alessandro, 47

Marchetti, E., 4F

Marchiori, Gianpietro, $1 \mathrm{~J}$

Marconi, A., 3R

Marcuzzi, Enrico, $1 \mathrm{~J}$

Mardones, Diego, $1 G$

Marini, D. L. R., 4P

Marisaldi, M., 31

Markoff, S., 31

Marquarding, Malte, 11
Marshall, Daniel, 4A

Marshall, Robert, 10

Marshall, Stuart, 09

Marson, Ralph, 04

Martí Ribas, J., 31

Martin, Adrian, 1X, 2A

Martinetti, E., 2C

Marty, L., 4F

Mason, Jon P., 3I

Mastrogiuseppe, Marco, 26

Mastropietro, Michele, 15, 2C, 3V

Masuda, Neal, 2M

Matsunaga, Noriyuki, 39

Mayer, Chris, OS

McBreen, Sheila, $1 \mathrm{~V}$

McCann, Kevin, 05

McCauliff, Sean, 3E

Medhi, Biman Jyoti, $4 \mathrm{O}$

Mégevand, Denis, 1T, 2H, 2K, 3Q

Melkumyan, David, 03, 3C

Merchant, Nirav, OF

Messina, Marco, OZ

Messineo, Rosario, 4D

Mi, W., OY

Middleton, Kevin F., 1X, 2X

Mighell, Kenneth J., OL, 11

Migoni, C., OB, 10

Miller, Joseph B., 1Q

Mills, David, 09, 20

Mineo, Teresa, 2E, 2R

Mito, Hiroyuki, 39

Mitsuda, Kazuma, 39

Miyata, Takashi, 39

Mizumoto, Yoshihiko, 30

Molfese, C., $4 \mathrm{~F}$

Molgó, Jordi, 1R, 2F, 3P

Molinari, Emilio, 1X, 2A

Molinari, Sergio, $\mathrm{OH}$

Molinaro, Marco, OH, 28, 44

Monson, Andrew, 38

Montmessin, F., 4F

Moore, Anna M., OT, 4A

Moore, Josh, 24

Moore, Liz, 07

Moore, William J., 24

Mora, Matias, ID

Morales-Muñoz, Rafael, 34, 3 I

Morgan, Edward, 3E

Morgenstern, A., 03

Mori, Kiyoshi, 39

Mori, Yuki, 39

Morii, Mikio, 39

Moritani, Yuki, 3B

Morokuma, Tomoki, 39

Morzinski, Katie M., OF

Moschetti, M., 3Q

Motohara, Kentaro, 39

Mugnuolo, R., 4F

Mundell, C., 31

Muñoz, Roberto, OW 
Murowinski, Rick, 4N

Nakada, Yoshikazu, 39

Nanni, M., 2D

Natarajan, Swaminathan, 02, 0K, 41

Navarrete, Julio, 14

Neefs, Eddy, 3l

Neilsen, E., OP

Nelson, Matthew, 38

Neyroud, N., 31

Nicol, Mark, 02, 0Z, 47

Nicolini, G., 4L

Nielsen, Jon G., $2 Q$

Nishioka, Hiroaki, 08

Noda, K., 31

Norris, Patrick, OL, 11

Nucciarelli, Giuliano, $1 \mathrm{~J}$

Nyman, Lars-Ake, 3W

Ó hÓgáin, Fionn, $1 \mathrm{~V}$

O'Brien, Alan, 12

O'Brien, P., 31

O'Callaghan, Derek, IV

Oggioni, Luca, 3Q

Ohsawa, Ryou, 39

Okumura, Shin-ichiro, 39

Olsen, Knut, OL, 11

Omar, Amitesh, 40

O'Mullane, William, $1 \mathrm{~V}$

Ong, R., 31

Onozato, Hiroki, 39

Onyuksel, Cem, 1Q

Orlati, Andrea, OB, OZ, 10, 2D

Osawa, Kentaro, 39

Ottensamer, R., $2 \mathrm{~W}$

Ovando, Nicolas, OD, 20

Owen, Russell, OG

Oya, Igor, 03, 0X, 3C

Paat, Anthony, 10

Pancrazzi, M., 4L

Pandey, Shreya, 41

Parejko, John, OG

Pareschi, Giovanni, 1J, 3L, 40

Pariani, G., 3Q

Parra, J., 20

Pasian, Fabio, 1B

Pastor-Morales, M. Carmen, 3

Patel, Manish R., 3l

Patel, Nimesh A., 08

Patil, Mangesh, 02

Patwari, Puneet, 41

Paxson, Charles, $1 Q$

Pecoraro, Marco, 4D

Pellegrin, F., OE

Pepe, F., 3Q

Perez, Jesus, 3J, 3K

Pessemier, Wim, 43

Petrucci, P. O., 31

Piascik, Andrzej S., 17

Piazzo, Lorenzo, 26

Pietriga, Emmanuel, OW, OX

Pietrowicz, Steve, 09
Pirrotta, S., 4F

Plummer, David A., 1Q

Poppi, S., OB, 10

Porter, Dallan, $1 \mathrm{~K}$

Power, Jennifer, 49

Pramskiy, Alexander, 36

Primet, Romain, OW

Probst, Ronald, 10

Przybilla, Norbert, 27

Punch, Michael, 3C

Quirrenbach, A., 34

Raguso, Maria Carmela, 26

Ramalingam, Balaji, 24

Ramos Mas, J. L., 42

Ramos, Francisco, 2F, 3P

Ramsey, Jason, 46

Ramsey, Larry, 38

Rao, Pramesh, 40

Raskin, Gert, 43

Ratzka, Thorsten, 27

Ravanmehr, Reza, 1L, 2T, 3U

Reardon, K., $1 \mathrm{~F}$

Reetz, Kristin, 10

Reil, K., OP

Reiners, A., 34

Reveco, Johnny, 23

Ribas, Ignasi, 2V, 34

Ribó, M., 31

Riccio, Giuseppe, $\mathrm{OH}$

Richard, P., 4G

Riddle, Reed L., OT, 4A

Ridgway, Stephen T., OL, II

Riera-Ledesma, Jorge, 2P

Riffeser, Arno, 2G

Riggi, Simone, 3S

Righini, S., OB, 10, 2D

Rinehart, Stephen, 3E

Rippa, Mathew J., 2M

Ristic, Bojan, 3l

Riva, Alberto, 4D

Riva, M., 2H, 2K, 3Q

Rizzi, A., 4P

Robertson, Paul, 38

Robles-Muñoz, Nicolás F., 3l

Roby, W., OY

Rodriguez, J., 31

Rodríguez-Gómez, Julio F., 3

Rodríguez-Losada, Jose A., 1R, 2F

Rogers, Kevin, $2 \mathrm{X}$

Romano, Patrizia, 2R, 31

Romoli, M., 4L

Rothmaier, Florian, 36

Roy, Arpita, 38

Rozbicki, Emil, 24

Russo, Federico, 3L, 3X, 40

Russo, Francesco, 3T

Rustici, Gabriella, 24

Sabater, Josep, 2P

Sadeh, I., OX

Saey, Philippe, 43

xviii

Proc. of SPIE Vol. $9913991301-18$ 
Saez, Alejandro, OD, 3J, 3K

Saez, Norman, OD

Saha, Abhijit, OL, 1 ।

Sahu, Sanjit, 40

Sako, Shigeyuki, 39

Saldias, Christian, 20

Sánchez, B., 4K

Sanderfer, Dwight, 3E

Sangiorgi, Pierluca, 3G, 3L, 3T, 3X, 40

Santin, $\mathrm{P} ., 2 \mathrm{H}, 2 \mathrm{~K}$

Sanz, Josep, 2V

Sanz-Mesa, Rosario, 31

Sarazin, Marc, 14

Sarugaku, Yuki, 39

Sato, Mikiya, 39

Satorre, Christophe, OU

Sauvage, Marc, 1B

Scagnetto, Ivan, 28

Schaefer, Christoph E. R., 35

Schaffenroth, Veronika, 27

Schellart, Pim, OG

Schiller, J., 34

Schindler, Karsten, 07

Schipani, P., 4F

Schisano, Eugenio, $\mathrm{OH}$

Schlichter, Jörg, 2G, 3M, 30

Schmid, Erich, 1A

Schmid, J., 31

Schubert, Kiaina, 2B

Schumacher, Germán, 09, 1W, 20

Schüssler, F., 31

Schwab, Christian, 38

Schwanke, Ullrich, 03, 31, 3C

Schwarz, Joseph, 03, 0X, 1D, 31, 3C, 3L, 40

Sciacca, Eva, $\mathrm{OH}$

Scuderi, Salvatore, 1J, 3L, 3X, 40

Sebag, Jacques, 09

Segreto, Alberto, 3T

Seifert, W., 34

Selvy, Brian, 09

Sepulveda, Jorge, OD

Serrano, S., OP

Serre, N., 31

Shalchian, Hengameh, $3 \mathrm{U}$

Shang, Zhaohui, OM

Shen, Tzu-Chiang, OC, OD, 23

Shen, Zhiqiang, 06

Shetrone, Matthew, 38

Shigeyama, Toshikazu, 39

Shimono, Atsushi, 3B

Shortridge, Keith, OU

Sick, Jonathan, OG

Sickafoose, Amanda, OR

Simard, LUC, 4A

Slabber, Martin, OA

Slozar, A., OP

Smareglia, Riccardo, 02, OH, 28, 2D, 2J, 3S, 44

Smedley, Scott, OU

Smith, Jeffrey C., 3E

Smith, Malcolm, 4J
Smith, Robert J., 17

Smith, Roger, OT

Snigula, Jan M., 2 G

Sohn, Ji Man, OT, 4A

Sol, H., 31

Sola, Igor S., 49

Solar, Mauricio, $1 \mathrm{G}$

Sommer, Heiko, 13, 14

Soto, Christian, 10

Soto, Ruben, OD

Sottile, Giuseppe, 3T

Sousa, Sérgio, $1 \mathrm{~T}$

Soyano, Takao, 39

Spadaro, D., 4L

Spiess, DJ, $1 \mathrm{~F}$

Sponza, M., 2D

Sprayberry, David, 10

Stagni, M., 2D

Staig, Tomás, OD, 1D

Stamerra, A., 31

Steele, lain A., 17

Stefansson, Gudmundur Kari, 38

Stilz, Ingo, 36

Stobie, Elizabeth B., OL, 11

Stolarczyk, T., 31

Straus, T., 4L

Stringhetti, LuCa, 1 J, 3L

Stuik, Remko, 2X

Subramaniam, Annapurni, 19

Summers, David, 10

Summers, Douglas M., 1Z, 49

Summers, Kellee R., $1 \mathrm{Z}$

Sun, $\mathrm{He}, 37$

Sun, Jinghai, $1 M$

Sundararaman, Harini, 09

Suzuki, Nao, 3B

Suzuki, Ryuji, OT, 4A

Swade, Daryl, 3E

Swain, Melanie A., 01

Swaminathan, N. 41

Swart, Paul, 02, OK

Swedlow, Jason R., 24

Swinbank, John, OG

Szeto, Kei, 4N

Tacchini, A., 2C, 21

Tait, Philip, 2B

Takahashi, Hidenori, 39

Takato, Naruhisa, 3B

Tamura, Naoyuki, 3B

Tan, Chengmin, 3A

Tanaka, Masaomi, 39

Tanci, Claudio, 1J, 2C, 2I, 3C, 3L, 3X, 40

Taniguchi, Yuki, 39

Tanikawa, Ataru, 39

Tarkowska, Aleksandra, 24

Tarusawa, Ken'ichi, 39

Taylor, Philip, is

Tenenbaum, Peter, 3E

Terrien, Ryan, 38

Testa, Vincenzo, 3L 
Theron, S., 4G

Thiebaut, C., 4G

Thomas, Ian R., 31

Thompson, Roger S., 02, $0 Z$

Toland, Trevor G., OQ

Toledo, Ignacio, 3W

Tollerud, Erik J., OG

Tominaga, Nozomu, 39

Torra, Jordi, 2P

Torres, Santiago, $2 \mathrm{P}$

Tosti, Gino, 03, 1J, 31, 3C, 3L, 3X, 40

Totani, Tomonori, 39

Trager, Scott C., 1X, 2X

Tran, Hien D., Ol

Trifoglio, Massimo, 2C, 2I, 3G, 3L, 40

Trivedi, Vatsal, 2L

Trujillo, Chadwick, 2M

Tsubota, Kevin, 05

Twicken, Joseph D., 3E

Uddin, Wahab, 40

Ukita, Nobuharu, 30

Urakawa, Seitaro, 39

Usui, Fumihiko, 39

Vagg, Daniel, IV

Valame, Snehal, $\mathrm{OZ}$

Vallenari, Antonella, 1X, 2X

Van den Heever, Lize, 02, 0A

van Gend, Carel, OR

Van Winckel, Hans, 43

Vandaele, Ann Carine, 31

Vandenbrouck, J., 31

Vanderbei, Robert, 37

Vanderspek, Roland, 3E

Vattiat, Brian, 46

Veillet, Christian, 49

Vela Nuñez, M., $2 Z$

Velez, Gaston, OD

Vera Sequeiros, Ignacio, 14

Véran, Jean-Pierre, 4J

Vercellone, Stefano, 31, 3L, 40

Vergani, S., 31

Verma, Khushbu, 1D

Vermeulen, Tom, 4N

Vico, I., 34

Vilardell, Francesc, 2V, 34

Vitello, Fabio, $\mathrm{OH}$

Vrcic, Sonja, 02, 2 Z

Vuerli, Claudio, 1B

Vuong, Minh V., OU

Walczysko, Petr, 24

Walsh, Shane, 49

Walth, Gregory, OT, 4A

Walther, Craig A., 32

Wampler, Steve, OS

Wang, C. OY

Wang, Liang, 30

Wang, Lianqi, 4J

Wang, Shin-Ywan, Ol, OY

Wang, Xiaofan, $3 \mathrm{~A}$

Wang, Zhong, $1 \mathrm{G}$
Watanabe, Junichi, 39

Watson, F., $1 \mathrm{~F}$

Wegner, Michael, 2G, 3M

Wegner, Peter, 03, 3C

Weinstein, Amanda, 3C

Weiss, Jason, OT, 4A

Wheeler, Caleb H., OQ

Whiting, Matthew, 11

Whittal, Hamish, OR

Wiant, Scott, IF, IP

Williams, Doug, 10

Williams, Eleanor, 24

Williams, Stewart J., 02, 0Z, 2L

Wilson, C., 4F

Wincentsen, James, 4A

Winkler, Roland, 36

Wischnewski, Ralf, 3C

Withington, Kanoa, 4N

Wolf, Jürgen, 07

Wright, Shelley A., OT, 4A

Wu, X., OY

Wu, Ya-Lin, OF

Yabe, Kiyoto, 3B

Yadava, Shobhit, 40

Yamaguchi, Jumpei, 39

Yamasaki, Chris, 2M

Yanagisawa, Kenshi, 30

Yang, Xiao, 3A, 3 Z

Yasuda, Naoki, 3B

Ye, Qian, 06

Yerli, Sinan K., 33

Yeşilyaprak, Cahit, 33

Yoshikawa, Makoto, 39

Young, Michael D., 18, 1H, 4E

Young, Peter J., 2Q

Zacchei, Andrea, 1B

Zago, Lorenzo, 33

Zanichelli, A., 2D

Zanmar Sanchez, Ricardo, 40

Zech, A., 31

Zechmeister, M., 34

Zeilinger, Werner W., 27

Zhang, Lijun, OY

Zhao, Cui, 3A

Zhu, Lichun, $1 \mathrm{M}$

Zoli, Andrea, 31, 3G, 3L

Zorba, Sonia, 44 


\section{Conference Committee}

Symposium Chairs

Colin Cunningham, UK Astronomy Technology Centre

(United Kingdom)

Masanori lye, National Astronomical Observatory of Japan (Japan)

Symposium Co-chairs

Allison A. Barto, Ball Aerospace \& Technologies Corporation

(United States)

Suzanne K. Ramsay, European Southern Observatory (Germany)

Conference Chairs

Gianluca Chiozzi, European Southern Observatory (Germany)

Juan C. Guzman, Commonwealth Scientific and Industrial Research

Organisation (Australia)

Conference Program Committee

Alan Bridger, UK Astronomy Technology Centre (United Kingdom)

Tom Donaldson, Space Telescope Science Institute (United States)

Jorge F. Ibsen, European Southern Observatory (Chile)

Kim K. Gillies, Thirty Meter Telescope Observatory Corporation

(United States)

José M. Filgueira, GMTO Corporation (United States)

Bret Goodrich, National Solar Observatory (United States)

Shui Hung Kwok, W. M. Keck Observatory (United States)

Richard M. Prestage, National Radio Astronomy Observatory

(United States)

David L. Terrett, RAL Space (United Kingdom)

Lize Van den Heever, SKA South Africa (South Africa)

Session Chairs

1 Project Overviews and Progress I

Gianluca Chiozzi, European Southern Observatory (Germany)

Richard M. Prestage, National Radio Astronomy Observatory

(United States) 
2 Telescope Control I

David L. Terrett, STFC Rutherford Appleton Laboratory

(United Kingdom)

José M. Filgueira, GMTO Corporation (United States)

3 Software Quality and Testing

Alan Bridger, UK Astronomy Technology Centre (United Kingdom)

Jorge Ibsen, ALMA (Chile)

4 Data Management and Archives I

Juan Carlos Guzman, Commonwealth Scientific and Industrial Research Organisation (Australia)

Jorge Ibsen, ALMA (Chile)

5 Cyberinfrastructure

Gianluca Chiozzi, European Southern Observatory (Germany)

Jorge Ibsen, ALMA (Chile)

6 Instrumentation Control

David L. Terrett, STFC Rutherford Appleton Laboratory

(United Kingdom)

Lize Van den Heever, SKA South Africa (South Africa)

$7 \quad$ UI/Web Technologies

Shui Hung Kwok, W. M. Keck Observatory (United States)

José M. Filgueira, GMTO Corporation (United States)

Lightning Talks

Alan Bridger, UK Astronomy Technology Centre (United Kingdom)

Shui Hung Kwok, W. M. Keck Observatory (United States)

Kim Gillies, Thirty Meter Telescope (United States)

Jorge Ibsen, ALMA (Chile)

8 Project Overviews and Progress II

Jorge Ibsen, ALMA (Chile)

Richard M. Prestage, National Radio Astronomy Observatory

(United States)

9 Data Processing and Pipelines I

Kim Gillies, Thirty Meter Telescope (United States)

Juan Carlos Guzman, Commonwealth Scientific and Industrial Research Organisation (Australia) 
10 Project Management

Bret D. Goodrich, National Solar Observatory (United States)

Richard M. Prestage, National Radio Astronomy Observatory

(United States)

11 Data Management and Archives II

Kim Gillies, Thirty Meter Telescope (United States)

Bret D. Goodrich, National Solar Observatory (United States)

12 Telescope Control II

Shui Hung Kwok, W. M. Keck Observatory (United States)

Lize Van den Heever, SKA South Africa (South Africa)

13 Software Engineering

José M. Filgueira, GMTO Corporation (United States)

David L. Terrett, STFC Rutherford Appleton Laboratory (United Kingdom)

14 Data Processing and Pipelines II

Alan Bridger, UK Astronomy Technology Centre (United Kingdom)

Shui Hung Kwok, W. M. Keck Observatory (United States)

Conclusion

Juan Carlos Guzman, Commonwealth Scientific and Industrial Research Organisation (Australia)

Gianluca Chiozzi, European Southern Observatory (Germany) 
Proc. of SPIE Vol. $9913991301-24$

Downloaded From: https://www.spiedigitallibrary.org/conference-proceedings-of-spie on 26 Apr 2023 Terms of Use: https://www.spiedigitallibrary.org/terms-of-use 